\title{
Attitudes face aux mathématiques: filles et garçons égaux dans la façon d'aborder leurs apprentissages?
}

\section{Philippe A. Genoud, Gabriel Kappeler et Matthias Guillod}

Cet article propose une analyse des attitudes socio-affectives des filles et des garçons face à leurs apprentissages en mathématiques durant leur scolarité post-obligatoire (gymnase). Par le biais d'un questionnaire auto-rapporté évaluant différentes perceptions qu'ont les élèves dans cette discipline (utilité perçue, sentiment de compétence, contrôlabilité), leurs affects (positifs, négatifs, ainsi que le sentiment de pouvoir les réguler), mais également la présence chez eux du stéréotype de masculinité des maths, nous avons pu dégager six profils caractéristiques. Ces derniers sont non seulement répartis inéquitablement selon le genre mais présentent des différences en termes d'investissement. De tels résultats questionnent sur l'impact des attitudes sur la motivation et les résultats des élèves (notamment les cercles vicieux qui s'instaurent), mais surtout ensuite sur l'orientation professionnelle de ces adolescents aux portes des universités et hautes écoles.

\section{Introduction}

La question de l'égalité entre femmes et hommes au niveau scolaire et professionnel a été source de nombreuses études depuis plusieurs décennies (Eagly, Eaton, Rose, Riger \& McHugh, 2012). Cependant, la sous-représentation des femmes au sein de carrières scientifiques - généralement dans les domaines des sciences, des techniques, des mathématiques et de l'informatique - reste une question d'actualité (e.g. Wyss, Huelskamp \& Siebert, 2012). En Suisse, la part des femmes diplômées dans ces domaines n'atteignait que 25\% en 2008 (Koller $\&$ Meffre, 2013). Bien que certaines études soulignent que les différences de performances en mathématiques tendent à se réduire (Lindberg, Hydemari, Petersen \& Linn, 2010), on constate toujours ces différences relatives au genre ${ }^{1}$ (Stoet \& Geary, 2012).

Les stéréotypes véhiculés dans la société sont souvent mis en avant pour comprendre ces différences de performances, que ceux-ci soient explicites (Spencer, Steele \& Quinn, 1999) ou implicites (Lane, Goh \& Driver-Linn, 
2012). Par le biais de facteurs personnels et sociaux tels la pression de la conformité ou encore les croyances relatives au genre (Leaper, Farkas \& Brown, 2011), l'attente de réussite et la valeur qui lui est attribuée (Eccles \& Wigfield 2002) semblent être au cœur de la compréhension du phénomène.

Outre les stéréotypes genre et leurs corollaires, l'état affectif de l'élève (qui se trouve lui-même parfois influencé par ces stéréotypes) joue un rôle non négligeable dans la mobilisation des ressources durant les apprentissages (Pekrun, Elliot \& Maier, 2009). Les émotions négatives que certains d'élèves ressentent en cours de mathématiques sont aussi réparties inéquitablement selon le genre, les filles étant plus souvent que les garçons perturbées par une forte anxiété (Devine, Fawcett, Szücs \& Dowker, 2012).

Ainsi, ce sont non seulement les croyances (ou représentations) de l'élève, mais aussi son ressenti affectif et son comportement (dimension conative) qui caractérisent ses attitudes face à l'apprentissage des mathématiques (Triandis, 1971; Fishbein \& Ajzen, 1975). En effet, le terme attitude peut être défini comme un «état interne à l'individu, résultant de la combinaison de perceptions, de représentations, d'émotions, d'expériences, et de l'analyse de leurs résultats. Cet état interne rend plus ou moins probable un comportement déterminé dans une situation donnée.» (Raynal \& Rieunier, 2001, p. 45). Ce concept permet en effet de mieux comprendre la façon dont les élèves appréhendent les mathématiques, même si les liens avec la réussite dans le domaine n'est pas toujours très important en termes de taille d'effet (Ma \& Kishor, 1997) et si la causalité ne se révèle pas être forcément dans le sens attendu (Ma \& Xu, 2004).

En reprenant chaque registre constitutif des attitudes, nous pouvons formuler certaines hypothèses concernant les différences selon le genre. En effet, de nombreuses publications - principalement liées à l'étude de la motivation en contexte scolaire - soulignent les différences entre filles et garçons dans l'approche de leurs apprentissages. Ces écarts ne concernent généralement pas les degrés préscolaires (Patrick, Mantzicopoulos, Samarapungavan \& French, 2008), mais apparaissent progressivement au cours de la scolarité et sont donc marqués à l'adolescence (Gurtner \& Genoud, 2006). Nous reprenons ci-dessous de manière plus détaillée ces différents travaux en explicitant leur articulation avec les dimensions constitutives des attitudes des élèves.

\section{Registre cognitif}

Dès le début de la scolarité primaire, les élèves se forgent des représentations sur leurs propres compétences. Basé sur un échantillon de plusieurs milliers d'élèves issus de contextes socioculturels variés, Stetsenko, Little, Gordeeva, Grasshof et Oettingen (2000) observent un biais de perception systématique chez les filles. À compétence supérieure, ces dernières évaluent leurs compétences de manière similaire aux garçons. Un tel pattern se retrouve chez les préadolescents (Malmberg \& Little, 2007), l'écart se creusant encore durant l'adolescence (Chen, Chen, Lee, Chen \& Keith, 2013). Même si le sentiment de compé- 
tence tend à baisser au fil de la scolarité (Wigfield, Eccles, Mac Iver, Reuman \& Midgley, 1991), de tels résultats sont systématiquement mis en évidence dans les recherches dans le domaine (e.g. Marsh \& Yeung, 1998; Nagy, Watt, Eccles, Trautwein, Lüdtke \& Baumert, 2010). Ainsi, nous postulons que dans notre échantillon, les filles rapporteront un sentiment de compétence en maths inférieur à celui des garçons.

L'utilité perçue des mathématiques est également une dimension appartenant au registre cognitif. Étroitement liée à la motivation extrinsèque du modèle de Deci et Ryan (1985), elle fait référence à la valeur instrumentale à long terme que l'élève donne aux différentes tâches liées à l'apprentissage d'une discipline (en l'occurrence les maths) pour des buts futurs tels la formation ou le métier envisagés (Eccles \& Wigfield, 2002). La présence de différences genre sur cette dimension est controversée. Alors que certaines études soulignent des écarts (augmentant avec l'âge) en faveur des garçons (e.g. Fredricks \& Eccles, 2002), d'autres non (e.g. Watt, 2004; Chouinard, Karsenti \& Roy, 2007). Il faut mentionner que cette dimension est en lien avec les objectifs professionnels futurs (ou utilité distale; Simons, Dewitte \& Lens, 2004) et qu'un effet d'interaction existe entre le genre et le souhait de se diriger vers des carrières en lien avec les maths (Watt, 2006). Les filles qui visent ce type de profession considèrent les maths comme extrêmement utiles, alors que leurs pairs masculins, dans la même situation, indiquent une utilité bien plus modérée. Malgré cela, et en se basant sur le fait que le choix de carrière dans le domaine est clairement l'apanage des garçons (Koller \& Meffre, 2013), nous faisons l'hypothèse que les garçons percevront d'avantage l'utilité des maths que les filles.

\section{Registre affectif}

De manière générale et pour l'ensemble des disciplines scolaires, même celles dans lesquelles elles réussissent mieux que les garçons, les filles démontrent une anxiété plus élevée vis-à-vis du travail et des évaluations scolaires (Martin, 2004; Marsh, Martin \& Cheng, 2008; Genoud, Ruiz \& Gurtner, 2009), même si les niveaux d'anxiété peuvent varier fortement entre les disciplines (Wolters $\&$ Pintrich, 1998). Le domaine des maths ne fait pas exception à la règle puisque les filles ont systématiquement des scores supérieurs en ce qui concerne l'anxiété (Devine et al., 2012; Chen et al., 2013), mais aussi d'autres affects négatifs tels le désespoir ou la honte (Frenzel, Pekrun \& Goetz, 2007).

Le nombre de publications présentant des outils évaluant l'anxiété des élèves en maths atteste bien de son importance dans cette discipline. De plus, les résultats concernant les différences selon le genre vont sans exception dans la même direction. L'intérêt des chercheurs pour les émotions positives est par contre clairement plus faible. Au niveau des outils d'évaluation, certains visent à distinguer différentes émotions positives tels le plaisir, le soulagement, la fierté ou l'espoir (Pekrun, Goetz, Frenzel, Barchfeld \& Perry, 2011) - ceci bien que les liens empiriques observés entre ces dernières soient élevés - d'autres par 
contre en proposent une mesure unidimensionnelle (Bai, 2011; Lichtenfeld, Pekrun, Stupnisky, Reiss \& Murayama, 2012). Il faut également noter que plusieurs échelles de motivation intègrent, sous la dimension "attrait de l'école» ou «intérêt», la notion de plaisir ressenti dans les activités d'apprentissage (e.g. Ntamakiliro, Monnard, Gurtner, 2000; Smith, Davy \& Rosenberg, 2012), cette dimension se confondant partiellement avec la notion de motivation intrinsèque (Ryan \& Deci, 2000; Marsh et al. 2013).

Au niveau des différences genre, les publications soulignent que les garçons ont des scores plus élevés en ce qui concerne les émotions positives (Frenzel et al., 2007; Lichtenfeld et al., 2012), avec des tailles d'effets tout à fait similaires aux écarts concernant les émotions négatives. De plus, l'analyse des résultats de l'étude TIMMS relève de fortes corrélations entre le sentiment de compétence et les affects positifs, tant pour les sciences que pour les maths (Marsh et al., 2013). Ainsi, même si notre échantillon est constitué d'élèves en scolarité post-obligatoire, nous pouvons postuler l'hypothèse suivante: les filles rapporteront plus d'affects négatifs et moins d'affects positifs que les garçons.

Il nous semble important aussi de mentionner que les affects positifs ne sont pas une dimension opposée aux affects négatifs sur le même continuum. S'il est rare qu'un élève puisse éprouver conjointement des émotions positives et négatives, on peut trouver cependant un bon nombre d'élèves aucunement affectés par leurs apprentissages, d'où la pertinence de séparer les deux notions. Les corrélations entre émotions positives et négatives apparaissent du reste plutôt modérées (Pekrun et al., 2011).

$\mathrm{Au}$ quotidien, hommes et femmes n'utilisent globalement pas les mêmes stratégies de régulation des émotions (Nolen-Hoeksema, 2012). Dans le domaine scolaire (et en maths plus spécifiquement), des différences apparaissent également, les filles ayant non seulement davantage recours à des stratégies socio-émotionnelles que les garçons, mais déployant aussi de manière générale plus de stratégies de coping (De Corte, Depaepe, Op't Eynde \& Verschaffel, 2011) comme par exemple développer des auto-verbalisations positives ou encore laisser vagabonder son esprit pour échapper à l'anxiété que provoque la tâche. Toutefois, la focalisation sur les états émotionnels et les tentatives de régulation interferent avec l'engagement et la persistance dans les activités d'apprentissage (Boekaerts, 2007). De plus, si l'on distingue volontiers les stratégies utilisées pour diminuer ou différer les émotions négatives (affect repair) de celles ayant pour but de maintenir ou renforcer les émotions positives (up regulation) (Krohne, 2003), il faut souligner que c'est la première catégorie qui est principalement concernée en contexte scolaire. Ainsi, les difficultés de régulation affective sont étroitement en lien avec la présence (ou persistance) d'affects négatifs et nous faisons par conséquent l'hypothèse que les filles montreront plus de difficultés dans cette régulation que leurs pairs masculins. 


\section{Registre comportemental}

Les dimensions relatives au registre comportemental sont habituellement situées sur un continuum entre approche et évitement (Triandis, 1971), l'approche étant souvent opérationnalisée par une (auto-)évaluation des efforts et de l'investissement consentis pour les apprentissages. Durant l'adolescence, les filles disent investir plus d'efforts et se montrent plus persistantes que les garçons (Goodenow \& Grady, 1993; Ryan, 2001). En ce qui concerne les maths plus spécifiquement, et malgré un intérêt moindre, les filles disent consacrer plus d'effort dans l'apprentissage de cette discipline (Chen et al., 2013). Par le biais d'une recherche effectuée dans un contexte francophone, Chouinard et al. (2007) relèvent aussi cet apparent paradoxe en mettant en évidence que les filles, bien que se sentant moins compétentes que les garçons, ont des scores significativement plus élevés en ce qui concerne le niveau d'effort fourni.

À l'instar des différentes recherches citées ci-dessus, nous faisons l'hypothèse que les filles indiqueront s'investir davantage que les garçons dans l'apprentissage des maths. De plus, dans la mesure où les différentes facettes de la motivation fluctuent au cours de la scolarité (Gurtner \& Genoud, 2006), nous nous intéresserons à examiner - de manière transversale - les évolutions de l'investissement, du début à la fin de la scolarité secondaire II.

\section{Aspect normatif}

Dans le domaine scolaire, le stéréotype qui est très certainement le plus important, le plus stable et culturellement universel (Cvencek, Meltzoff \& Kapur, 2014) concerne la conception des maths comme domaine masculin. La compréhension des mécanismes de formation et de maintien de ce stéréotype met en évidence le rôle que jouent les parents et les enseignants (Gunderson, Ramirez, Levine \& Beilock, 2012) ainsi que l'individu lui-même au sein d'un contexte socio-structurel (Bussey \& Bandura, 1999) où de nombreux stéréotypes circulent. Indépendamment du bien-fondé de ce stéréotype (Kane \& Mertz, 2012), sa prise en compte dans les recherches explique dans une bonne mesure la participation et la réussite des élèves (Brodish \& Devine, 2009; Nosek et al., 2009; Lane \& al., 2012).

Ce stéréotype peut s'observer chez l'enseignant, notamment lorsqu'il a des répercussions marquées sur les évaluations des travaux des élèves (Lafontaine $\&$ Monseur, 2009). Toutefois, il est plus fréquemment étudié lorsqu'il est présent chez les élèves (stereotype threat; Spencer et al., 1999) et qu'il a un impact sur les performances et les orientations professionnelles des filles (Cheryan, 2012; Correll, 2001), notamment par le biais du sentiment de compétence. Ainsi, à l'instar des résultats relevés par Plante, de la Sablonnière, Aronson et Théorêt (2013) dans un contexte scolaire francophone (école secondaire), nous nous attendons à un effet d'interaction entre le genre et sentiment de compétence, plus précisément, à une corrélation positive pour les garçons mais négative pour les filles. 


\section{Profils d'attitudes}

Les liens entre les différentes facettes des attitudes s'avèrent relativement importants, et tout particulièrement ceux entre les aspects cognitifs et affectifs (Pekrun et al., 2011). En effet, les croyances et appréciations d'un individu ont un impact sur son ressenti. Si la théorie control-value (Pekrun, 2006) clarifie les différents types d'émotions générées par des attentes de résultats (prospectif) et par les réussites ou échecs effectifs (rétrospectif), l'auteur note également une circularité de la chaîne causale puisque les émotions vont à leur tour conditionner le sujet, ses attentes et ses croyances. De plus, si les différentes théories des émotions permettent de bien comprendre les processus sous-jacents à leurs apparitions, elles ne permettent pas d'y inclure un plus large spectre d'expériences affectives qu'un sujet peut ressentir en contexte (Op't Eynde, De Corte \& Verschaffel, 2001). Les différents modèles des attitudes possèdent donc des dimensions qui, au niveau théorique, se structurent de manière pertinente dans des modèles très cohérents mais qui, de manière empirique, peinent souvent à se distinguer nettement les unes des autres.

Bien que nous ayons un tableau relativement clair des différences genre sur nombre de dimensions relatives aux attitudes, il nous semble intéressant d'examiner si des profils d'élèves peuvent être dégagés et si certains de ces profils sont prédominants chez les garçons ou chez les filles. Nous visons donc une mise en évidence de types d'élèves particuliers regroupés par leur proximité sur les aspects cognitifs et affectifs de leurs attitudes. Dans notre démarche nous considérons, à l'instar d'autres travaux (e.g. Chouinard et al., 2007), le registre comportemental comme une variable critère du modèle.

\section{Méthodologie}

\section{Échantillon et passation}

La récolte de donnés a eu lieu dans quatre établissements scolaires du secondaire II (lycée) du canton de Fribourg dans le courant de l'automne 2013. Nous avons pris soin d'avoir dans chaque école des classes de tous les degrés ( $^{\mathrm{re}}$ année à $4^{\mathrm{e}}$ année) afin d'avoir un pourcentage similaire d'élèves de chaque année. Notre échantillon est constitué de 416 élèves (181 garçons et 235 filles) dont l'âge varie entre 15 et 21 ans $(\mathrm{m}=17.2 ; \mathrm{s}=1.4)$. Les questionnaires ont été distribués au sein des différentes classes par leurs enseignants habituels. Il a été demandé aux élèves de répondre à toutes les questions dans l'ordre, le plus spontanément possible (nous n'observons que $0.2 \%$ de réponses manquantes). Les élèves ont été informés de l'anonymat des questionnaires et les enseignants ont été priés d'éviter toute pression (p.ex. ne pas passer dans les rangs durant la passation) afin de ne pas biaiser les réponses. 


\section{Mesures}

Le Questionnaire des attitudes socio-affectives en mathématiques (QASAM; Genoud \& Guillod, 2014) est un instrument récent constitué de 45 items au total et évaluant différentes perceptions qu'ont les élèves relativement à leurs apprentissages en maths (utilité perçue, sentiment de compétence, contrôlabilité), à leurs affects (positifs, négatifs, ainsi que le sentiment de pouvoir les réguler) et à leurs comportements face à l'apprentissage (investissement). Il évalue également s'ils considèrent cette discipline comme un domaine masculin (voir tableau 1).

Tableau 1. Dimensions du Questionnaire des attitudes socio-affectives en mathématiques (QASAM), avec exemples d'items et alpha de Cronbach

\begin{tabular}{|llcc|}
\hline Dimensions & Exemple d'item & N & Alpha \\
\hline Registre cognitif & $\begin{array}{l}\text { Les maths me seront précieuses dans mon } \\
\text { Utilité }\end{array}$ & 5 & .80 \\
futur (formation et emploi). & & \\
Compétence & $\begin{array}{l}\text { J'ai beaucoup de potentiel dans le domaine } \\
\text { des maths. }\end{array}$ & 6 & .94 \\
Contrôlabilité & $\begin{array}{l}\text { Ma compréhension en maths dépend des } \\
\text { efforts que je fournis. }\end{array}$ & 5 & .74 \\
Registre affectif & $\begin{array}{l}\text { Je me réjouis de voir arriver l'heure de maths. } \\
\text { Affects positifs }\end{array}$ & 6 & .93 \\
Affects négatifs & $\begin{array}{l}\text { Je suis angoissé-e lorsque je fais mes devoirs } \\
\text { de maths. }\end{array}$ & 6 & .88 \\
Régulation affective & $\begin{array}{l}\text { Je maîtrise mon stress durant les évaluations } \\
\text { de maths. }\end{array}$ & 6 & .79 \\
Registre comportemental & $\begin{array}{l}\text { Je suis assidu-e et concentré-e durant le cours } \\
\text { de maths. }\end{array}$ & 6 & .83 \\
Investissement & $\begin{array}{l}\text { Les garçons sont à la base plus doués pour les } \\
\text { maths que les filles. }\end{array}$ & 5 & .86 \\
Mesure normative & 5 & 6 & \\
\hline
\end{tabular}

Le questionnaire est proposé sous une forme auto-rapportée et les participants doivent évaluer leur degré d'accord par rapport à chacune des affirmations (items) proposées sur une échelle de Likert à 6 niveaux allant de 0 (= Pas du tout d'accord) à 5 (= Tout à fait d'accord). 
L'instrument possède de bonnes caractéristiques psychométriques au niveau de la structure factorielle, de l'homogénéité interne des échelles et de la stabilité de la mesure, tant pour une population du secondaire II (post-obligatoire) que pour des élèves plus jeunes, dans les degrés du secondaire I (Genoud, Kappeler $\&$ Ebiner, 2014).

\section{Présentation des résultats}

Dans un premier temps, nous avons mené une analyse descriptive de la distribution des scores des différentes dimensions. Seule la dimension Masculinité présente une forte asymétrie positive (skewness $=1.18$; $\mathrm{SE}=.12$ ) où $30 \%$ des répondants n'adhèrent pas du tout à ce stéréotype (score minimal). Malgré cela, l'écart-type de cette distribution reste tout à fait comparable à celui des autres dimensions de ce questionnaire offrant ainsi une variabilité suffisante pour les différentes analyses envisagées.

Nous avons ensuite examiné les différences entre filles et garçons pour chacune des dimensions des attitudes prises séparément. Les tests du $t$ de Student mettent en évidence des différences significatives pour chaque dimension, à l'exception de la Contrôlabilité pour laquelle le score des garçons ne dépasse que trop faiblement celui des filles ( $c f$. Figure 1).

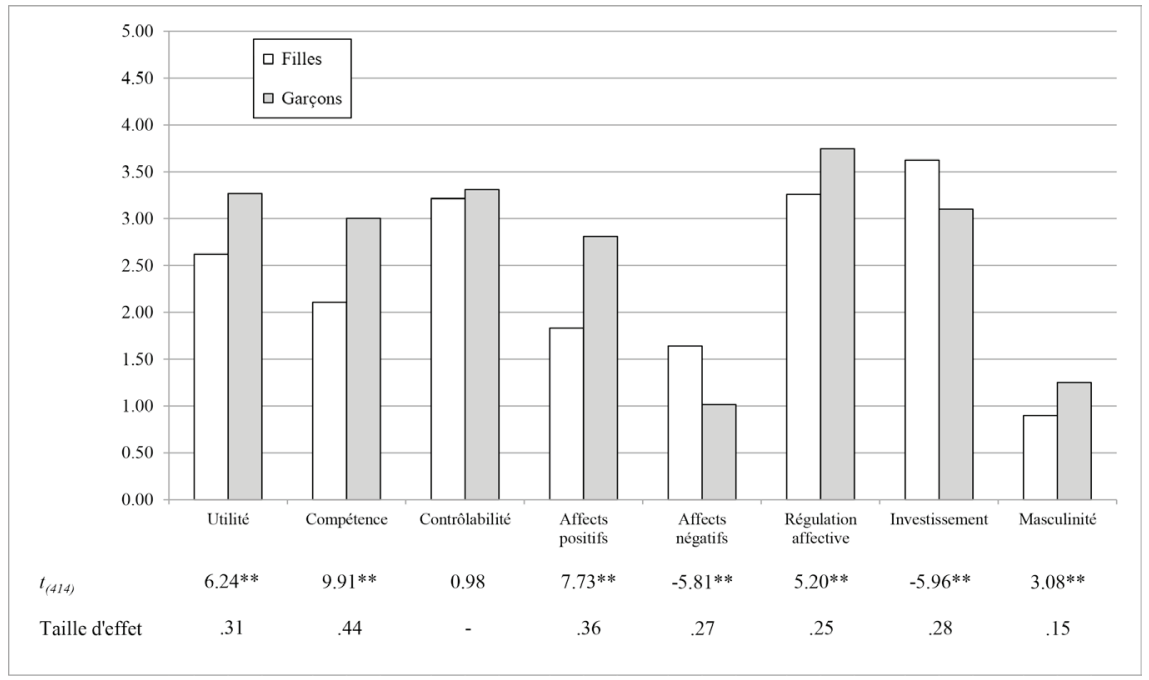

Note. Les tailles d'effet sont données par le coefficient de corrélation de Pearson et évaluées selon les critères de Cohen (Field, 2009): $r=.10$ (faible); $r=.30$ (moyen); $r=50$ (large). Les seuils de significativité sont de ${ }^{*} p<.05,{ }^{* *} p<.01$

Figure 1. Différences selon le genre sur chaque dimension des attitudes 
Les écarts les plus importants concernent les dimensions Compétences et Affects positifs pour lesquelles les garçons montrent des valeurs beaucoup plus élevées. De plus, comparativement aux filles, ils estiment les maths plus utiles pour leur futur, se sentent plus compétents dans leurs apprentissages, en retirent plus de plaisir tout en ressentant moins d'anxiété ou de stress, et disent mieux arriver à réguler leurs affects. Les filles indiquent quant à elles un investissement plus prononcé.

Au niveau temporel, nous avons observé l'évolution des différentes dimensions de manière transversale. Nous avons ainsi effectué des analyses de variance en tenant compte du degré scolaire des élèves, mais également du genre pour examiner d'éventuelles trajectoires différentes. Deux tests se révèlent significatifs. L'investissement des garçons diminue en cours de scolarité $\left(\mathrm{F}_{(3,408)}=2.85\right.$; $\mathrm{p}<.01)$ avec un score moyen de 3.36 en première année qui baisse progressivement jusqu'à 2.71 en quatrième année. La seconde analyse significative concerne le stéréotype de masculinité des maths $\left(\mathrm{F}_{(3.408)}=6.52 ; \mathrm{p}<.01\right)$ qui augmente très nettement entre la troisième et la dernière année chez les garçons et aussi - mais dans une moindre mesure - chez les filles.

Nous nous sommes également penchés sur les corrélations entre les dimensions du QASAM, et plus particulièrement sur les différences de corrélations selon le genre. Deux liens different significativement selon le genre. La corrélation Compétence-Masculinité est de .25 pour les garçons et de -.29 chez les filles. Outre la différence de signe, l'écart s'avère significatif $\left(t_{(410)}=5.58 ; \mathrm{p}<.01\right)$; le genre pouvant être considéré comme une variable modératrice $\left(\mathrm{F}_{\text {change }(1,412)}\right.$ $=32.14 ; \mathrm{p}<.01$; delta $\left.\mathrm{R}^{2}=7.1 \%\right)$. Il en va de même pour la corrélation entre les Affects positifs et la Masculinité où l'on trouve $r=.21$ pour les garçons et $\mathrm{r}=-.28$ chez les filles $\left(t_{(410)}=5.04 ; \mathrm{p}<.01\right.$; effet modérateur $\mathrm{F}_{\text {change }(1,412)}=$ $25.71 ; \mathrm{p}<.01$; delta $\left.\mathrm{R}^{2}=5.7 \%\right)$.

L'étape suivante de nos analyses a visé la constitution de groupes d'élèves ayant un profil similaire. Afin d'obtenir la plus forte variabilité inter-cluster et la meilleure similarité intra-cluster, nous avons donc opté pour une analyse de classification par la méthode des nuées dynamiques (K-means clustering). S’il existe un grand nombre de méthodes et d'algorithmes permettant de déterminer le nombre de clusters à retenir, aucun consensus ne semble émerger dans la littérature à ce sujet (Steinley $\&$ Brusco, 2011). De plus, les simulations aboutissent à des conclusions différentes en fonction de la taille de l'échantillon et du degré de complexité du vrai modèle (Lebarbier \& Mary-Huart, 2006). Ainsi, ces critères techniques ne sont généralement pas suffisants et une démarche heuristique d'interprétation des regroupements est nécessaire pour obtenir une solution pertinente. Nous avons donc opté pour une démarche en deux temps (Milligan, 1980). La première étape a consisté en une analyse de classification hiérarchique (méthode de Ward et calcul des distances euclidiennes au carré) permettant l'observation de l'agrégation des sujets. Sur cette base-ci, nous avons retenu trois solutions: 2,3 et 6 clusters. La seconde étape a reposé sur l'examen 
des analyses de classification non hiérarchique avec les trois solutions retenues préalablement, avec comme critères le nombre suffisant de sujets par groupe, la significativité des analyses de variances pour toutes les variables insérées dans de modèle, et la parcimonie de la solution.

Le modèle à 2 clusters distingue les élèves plutôt bons en maths de ceux qui ont des difficultés. La dimension Masculinité ne discrimine cependant pas suffisamment les deux groupes. Le modèle à 3 clusters intercale un groupe intermédiaire et présente le même défaut. Le modèle à 6 clusters $\left(\eta^{2}=.56\right)$ s'est avéré le plus pertinent. Afin d'en faciliter la lecture, nous les avons répartis en trois groupes de deux (voir Figure 2).

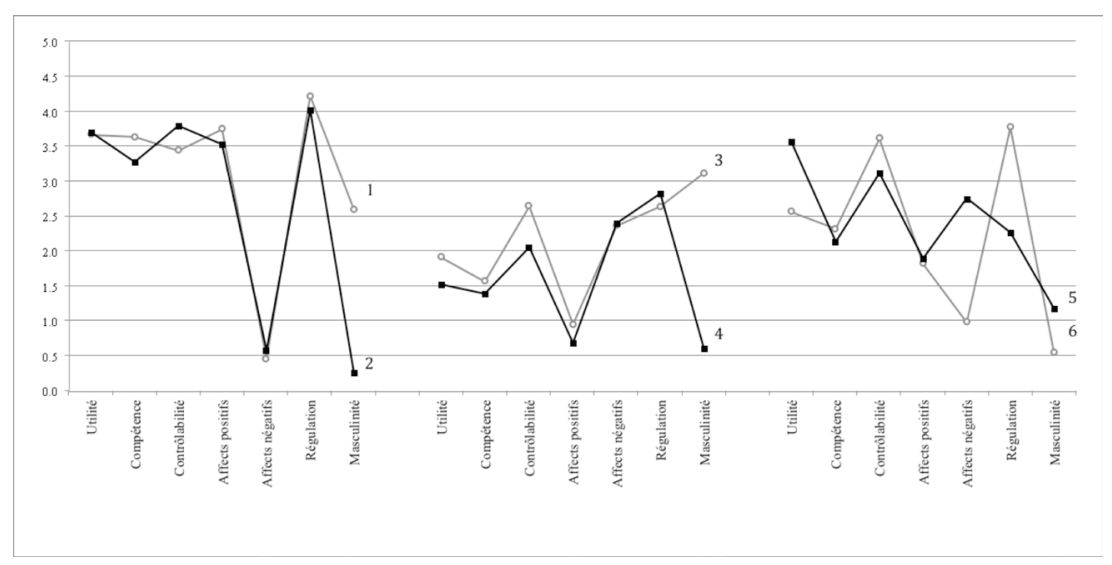

Figure 2. Profil des 6 clusters (cluster 1: $N=55$; cluster 2: $N=102$; cluster 3: $N=35$; cluster 4: $N=64$; cluster 5: $N=51$; cluster $6: N=109$ )

Les deux premiers clusters (dans lequel on trouve 56\% des garçons) correspondent à des profils d'élèves qui ont de la facilité en maths. Ils different uniquement dans le stéréotype masculinité. Au niveau de la répartition selon le genre, le cluster 1 regroupe presque exclusivement des garçons (91\%), alors que le cluster 2 est équilibré (51\% de garçons). Les deux clusters suivants (3 et 4) présentent des profils inverses. Ce sont des élèves qui apparaissent plutôt en difficulté. À nouveau, ces clusters se distinguent par le score de masculinité des maths et sont cette fois-ci composés majoritairement de filles ( $80 \%$ dans le cluster 3 et $78 \%$ dans le cluster 4 ). Le cluster 5 est un profil intermédiaire (sentiment de contrôlabilité modéré malgré une compétence perçue relativement basse) qui présente cependant un score marqué pour les affects négatifs ainsi qu'une régulation des affects plutôt faible (la plus faible de tous les profils); on n'y trouve aucun déséquilibre dans la proportion filles/garçons. Finalement, le cluster 6 dans lequel se trouve un quart des élèves - présente un profil caractérisé quant à lui par la faible présence d'affects (positifs et négatifs), mais une très bonne 
régulation affective tout comme un fort sentiment de contrôle. Toutefois, c'est le score d'utilité et de compétence perçue qui se révèle être moyen. Au niveau inférentiel, on retrouve sans surprise une répartition hétérogène des profils selon le genre avec un $\chi^{2}(5)=76.35(\mathrm{p}<.01)$.

Nous avons examiné les différences d'investissement entre les élèves des six clusters trouvés. L'analyse de variance réalisée se révèle significative $\left(\mathrm{F}_{(5,411)}=\right.$ 3.39; $\mathrm{p}<.01$ ). Les analyses post-hoc (procédure de Tukey) indiquent une seule différence significative $(\mathrm{p}<.01)$ entre le cluster 1 et le cluster 2 : les élèves du premier ont le niveau d'investissement le plus faible de tous $(\mathrm{m}=3.00 ; \mathrm{SD}=$ 1.07) alors que ceux du deuxième fournissent le plus d'efforts pour l'apprentissage des maths $(\mathrm{m}=3.62 ; \mathrm{SD}=0.86)$.

\section{Discussion}

Dans les comparaisons des scores entre filles et garçons, nous observons comme attendu des différences prononcées pour plusieurs des dimensions mesurées, ces résultats allant dans le sens de nombreux travaux publiés dans le domaine. Différentes hypothèses sont par conséquent validées. En premier lieu, les filles mentionnent avoir un sentiment de compétence inférieur aux garçons. Il faut noter que ce sentiment n'est, de manière générale, pas directement en lien avec la réussite effective et/ou les notes attribuées par l'enseignant. Différentes publications analysent en effet les compétences en maths selon le genre, notamment celles se basant sur les résultats TIMSS. Durant la scolarité obligatoire, les garçons ont une performance légèrement supérieure aux filles (Marsh et al., 2013), constatation également valable pour la Suisse (Mariotta, 2011). Pour ce qui est du secondaire II, les données sont rares, mais il semble que l'écart observé en faveur des garçons se réduise progressivement jusqu'à l'âge adulte (Lindberg et al., 2010). Par conséquent, nous pouvons partir de l'idée que dans notre échantillon, filles et garçons ont certainement des compétences relativement similaires (notons que nous n'avons pas d'informations sur les évaluations faites par les enseignants) et que le sentiment de compétence differe selon le genre parce que (1) les filles sont plus critiques envers elles-mêmes et montrent moins d'auto-satisfaction que les garçons (Frey \& Rubble, 1987), (2) l'appréciation de leurs compétences est plus fluctuante dans le sens où leurs perceptions sont plus proches de leurs résultats effectifs (Bouffard, Marcoux, Vezeau \& Bordeleau, 2003), et (3) la présence d'un stéréotype genre influence de manière différentielle le sentiment de compétence, ici en défaveur des filles (Cvencek et al., 2014).

Notre deuxième hypothèse portant sur l'écart concernant l'utilité perçue des maths est également validée. Les garçons accordent plus d'utilité à cette discipline. Pour une grande partie des élèves, si le choix précis de cursus suite à la maturité n'est pas totalement arrêté, les domaines dans lesquels ils souhaitent évoluer professionnellement s'affinent clairement. Par conséquent, ce résultat 
peut se lire très simplement par l'orientation envisagée des élèves vers des formations et des carrières où les maths sont présentes (Sadler, Sonnert, Hazari \& Tai, 2012). Une question portant sur le type de profession (ou formation) envisagée aurait pu amener des éléments d'interprétation supplémentaires comme un effet modérateur sur la différence observée selon le genre.

Pour ce qui est des trois dimensions appartenant au registre affectif, nos résultats vont dans le sens des hypothèses qui sont dès lors vérifiées. Sans surprise, le lien entre la présence d'affects négatifs et un déficit dans la régulation des émotions s'avère très fort tant chez les filles $(r=-.77)$ que chez les garçons $(r=-.74)$; ce sont d'ailleurs les corrélations les plus fortes entre les dimensions du QASAM. Ce résultat confirme l'importance des compétences de régulation pour gérer et atténuer l'anxiété et le stress généré par l'apprentissage des maths et par les évaluations qui jalonnent le programme. La taille d'effet est un peu plus importante pour les affects positifs que pour les deux autres dimensions. Il faut encore noter qu'ils sont un près de $12 \%$ (dont $1 / 3$ de garçons et $2 / 3$ de filles) à n'éprouver que peu ou pas d'affects (positifs et négatifs).

La différence postulée concernant la dimension Investissement va elle aussi dans la direction attendue, la preuve empirique étant déjà étayée par de nombreux travaux antérieurs. Au secondaire II, les filles disent consacrer plus d'efforts et être plus assidues dans leurs apprentissages en maths. Malgré une relative stabilité des autres dimensions (registres cognitif et affectif), on observe dans nos données un déclin de l'investissement chez les garçons, ce qui creuse l'écart déjà présent en début du cursus. Dans la mesure où une diminution de la motivation est déjà observée durant le cursus obligatoire du secondaire I (Genoud et al., 2009), il serait intéressant d'observer de manière longitudinale la transition vers le secondaire II. Avec un écrémage au niveau de population et une éventuelle influence positive du changement de degré et d'établissement, on pourrait s'attendre à un sursaut important au niveau de la motivation des élèves, qui tend visiblement alors à s'éroder à nouveau au fil du temps.

L'interaction postulée entre le genre et le sentiment de Compétence dans l'explication de la présence sur stéréotype de Masculinité des maths est bien présente dans nos données empiriques. Ce dernier semble alors être un moyen pour les élèves d'expliquer différentiellement la présence de compétences (chez les garçons) respectivement de leur absence (chez les filles). Il peut dès lors être considéré comme une attribution causale par certains élèves pour expliquer leur niveau en maths, avec (selon la théorie de Weiner, 1985) un locus interne, stable et non contrôlable. Il est cependant difficile de déterminer si les stéréotypes apparaissent de manière préalable à la construction des représentations par l'élève de ses propres compétences (Brodish \& Devine, 2009) ou s'il apparait comme une justification déresponsabilisante a posteriori (e.g. Plante et al., 2013). Par conséquent, postuler une influence réciproque (cercle vicieux) semble tout à fait justifiable, d'autant plus chez les filles où une activation de ce stéréotype ne peut que pousser à un retrait et, conséquemment, à une baisse des performances renforçant le bien-fondé du stéréotype. 
Le stéréotype apparaît également comme un élément clef dans l'interprétation des différents profils. On y retrouve ainsi l'effet d'interaction par la présence d'un écart marqué sur cette dimension dans le groupe des bons élèves, respectivement chez ceux qui sont en difficulté. La forte présence d'une conception masculine des maths est presque exclusivement un trait des garçons dans le premier groupe et des filles dans le second. Il est intéressant de relever la différence d'investissement qui est étroitement liée à ce stéréotype chez les meilleurs élèves. Les deux derniers clusters ( 5 et 6 ) constituent deux groupes intermédiaires mais qui se différencient toutefois clairement dans leur profil. Le cluster 5 - que nous pourrions nommer profil «anxieux» - est constitué d'élèves conscients de l'utilité des maths et de l'impact de leur travail sur les résultats, mais qui se laissent envahir par le stress sans stratégies pour le contrôler. Le cluster 6 quant à lui est un profil «moyen»; il est caractéristique d'élèves qui ne se sentent pas forcément compétents et qui n'ont pas beaucoup de ressentis positifs et négatifs. C'est le profil qui se trouve le plus proche du score moyen de tous les élèves, ceci pour chacune des dimensions.

\section{Conclusion}

Le design méthodologique de notre recherche ne permettant pas de définir des causalités, nous avons pris le parti de considérer l'ensemble des mesures comme un «cliché» qui caractérise chaque élève dans sa façon de se positionner face aux mathématiques. Par le biais d'un questionnaire auto-rapporté évaluant les attitudes dans le domaine cognitif, affectif et comportemental, ainsi qu'un score mesurant la présence du stéréotype de masculinité des maths, nous avons pu récolter des données auprès d'élèves du secondaire II (cursus post-obligatoire).

Les diverses hypothèses portant sur les différences d'attitude selon le genre ont toutes été confirmées, ceci étant un gage de validité des mesures effectuées (validité hypothético-déductive). Si ces résultats ne semblent a priori pas forcément amener de nouvelles connaissances dans la mesure où de nombreuses publications scientifiques font déjà état de ces divergences, il faut préciser que la plupart d'entre elles se rapportent à des élèves en scolarité primaire et secondaire (I). Le fait de retrouver des patterns de résultats identiques auprès d'élèves plus âgés dans la formation post-obligatoire (où sont réunis uniquement ceux qui ont les meilleurs résultats) montre qu'avec le temps, aucun changement ne semble se dessiner.

Par le biais d'une analyse en cluster (6 profils d'attitude distincts), nous pouvons constater que le stéréotype selon lequel les maths sont une discipline pour les garçons explique plusieurs différences genre observées, notamment les efforts consentis pour les apprentissages chez les élèves qui ont le plus de facilité. Il faut cependant rappeler que ce stéréotype est totalement absent chez près d'un tiers des élèves et que la distribution de la variable est fortement asymétrique, 
sans que nous puissions évaluer la part de désirabilité présente dans les réponses des élèves.

Au final, les différents profils suggèrent des attitudes singulières dont certaines sont caractéristiques des garçons respectivement des filles. De tels résultats soulèvent donc la question de l'impact de telles représentations sur les choix de cursus et de carrière pour ces étudiants aux portes des universités et hautes écoles (Sadler et al., 2012), et surtout de la manière d'y faire face si l'on souhaite non pas forcément une égalité en terme de quotas, mais une égalité de chances d'accès, qui ne soit pas freinée par des croyances largement répandues, mais sans fondement.

Le travail à ce niveau-là est à faire de manière très précoce (Martinot, Bagès \& Désert, 2012) puisque les stéréotypes se cristallisent progressivement et ont un impact sur les performances, notamment par le biais des affects. Il doit toutefois se poursuivre tout au long du parcours scolaire puisque le contexte et les perceptions de l'élève évoluent. Le stéréotype de masculinité des maths semble avoir un impact sur les deux composantes du modèle expectancy-value de Eccles et ses collègues (Eccles, 2005); réduire son importance aux yeux des élèves pourrait améliorer les attentes de réussite en maths, l'utilité et la valeur perçues de l'apprentissage ainsi que le fait d'arriver à se projeter dans des carrières où les maths et autres disciplines associées sont primordiales.

\section{Note}

1 Il nous semble important de clarifier l'utilisation du terme "genre» dans cet article. Si le sexe fait référence à la caractéristique biologique, le genre est relatif à une distinction culturelle (Bereni, Chauvin, Jaunait \& Revillard, 2012). Dans la mesure où notre recherche porte clairement sur les rôles sociaux, ancrés culturellement et ayant une influence sur les attentes, nous assumons donc l'emploi de ce terme, bien que la mesure effective réalisée soit techniquement une évaluation de la caractéristique biologique (le sexe). En effet, on ne peut réduire à de simples causes biologiques les différences observées en matière d'attitudes et de stéréotypes par exemple.

\section{Références bibliographiques}

Bai, H. (2011). Cross-validating a bidimensional Mathematics Anxiety Scale. Assessment, 18(1), 115-122.

Bereni, L., Chauvin, S., Jaunait, A. \& Revillard, A. (2012). Introduction aux études sur le genre (2e édition). Bruxelles: De Boeck.

Boekaerts, M. (2007). Understanding students' affective processes in the classroom. In P.A. Schutz \& R. Pekrun (Ed.), Emotion in education (pp. 37-56). Oxford: Elsevier.

Bouffard, T., Marcoux, M.-F., Vezeau, C. \& Bordeleau, L. (2003). Changes in self-perceptions of competence and intrinsic motivation among elementary schoolchildren. British Journal of Educational Psychology, 73(2), 171-186.

Brodish, A. B. \& Devine, P. G. (2009). The role of performance-avoidance goals and worry in mediating the relationship between stereotype threat and performance. Journal of Experimental Social Psychology, 45(1), 180-185.

Bussey, K. \& Bandura, A. (1999). Social cognitive theory of gender development and differentiation. Psychological Review, 106(4), 676-713. 
Chen, H.-Y., Chen, M.-F., Lee, Y.-S., Chen, H.-P. \& Keith, T. Z. (2013). Gender reality regarding mathematic outcomes of students aged 9 to 15 years in Taiwan. Learning and Individual Differences, 26, 55-63.

Cheryan, S. (2012). Understanding the paradox in math-related fields: Why do some gender gaps remain while others do not? Sex Roles, 66(3-4), 184-190.

Chouinard, R., Karsenti, T. \& Roy, N. (2007). Relations among competence beliefs, utility value, achievement goals, and effort in mathematics. British Journal of Educational Psychology, 77(3), 501-517.

Correll, S. J. (2001). Gender and the career choice process: The role of biased self assessments. American Journal of Sociology, 106(6), 1691-1730.

Cvencek, D., Meltzoff, A. N. \& Kapur, M. (2014). Cognitive consistency and math-gender stereotypes in Singaporean children. Journal of Experimental Child Psychology, 117, 73-91.

De Corte, E., Depaepe, F., Op't Eynde, P. \& Verschaffel, L. (2011). Students's self-regulation of emotions in mathematics: An analysis of meta-emotional knowledge and skills. ZDM: The International Journal on Mathematics Education, 43(4), 483-495.

Deci, E. L. \& Ryan, R.M. (1985). Intrinsic motivation and self-determination in human behavior. New York: Plenum Press.

Devine, A., Fawcett, K., Szücs, D. \& Dowker, A. (2012). Gender differences in mathematics anxiety and the relation to mathematics performance while controlling for test anxiety. Behavioral and Brain Functions, 8(33), 1-9.

Eagly, A. H., Eaton, A., Rose, S. M., Riger, S. \& McHugh, M. C. (2012). Feminism and psychology: Analysis of a half-century of research on women and gender. American Psychologist, 67(3), 211-230.

Eccles, J.S. (2005). Studying gender and ethnic differences in participation in math, physical science, and information technology. New Directions for Child and Adolescent Development, 110, 7-14.

Eccles, J. S. \& Wigfield, A. (2002). Motivational beliefs, values, and goals. Annual Review of Psychology, 53, 109-132.

Fishbein, M. \& Ajzen, I. (1975). Belief, attitude, intention and behavior: An introduction to theory and research. Reading, MA: Addison-Wesley.

Fredricks, J. A. \& Eccles, J. S. (2002). Children's competence and value beliefs from childhood through adolescence: Growth trajectories in two male-sex-typed domains. Developmental Psychology, 38(4), 519-533.

Frenzel, A. C., Pekrun, R. \& Goetz, T. (2007). Girls and mathematics - A “hopeless" issue? A control-value approach to gender differences in emotions towards mathematics. European Journal of Psychology of Education, 22(4), 497-514.

Frey, K. S. \& Ruble, D. N. (1987). What children say about classroom performance: sex and grade differences in perceived competence. Child Development, 58(4), 1066-1078.

Genoud, P. A. \& Guillod, M. (2014). Développement et validation d'un questionnaire évaluant les attitudes socio-affectives en maths. Recherches en Éducation, 20, 140-156.

Genoud, P. A., Kappeler, G. \& Ebiner, J. (2014, juin). Les attitudes pour expliquer la réussite en maths: différences selon le contexte. Communication présentée Congrès annuel de la Société Suisse pour la Recherche en Éducation (SSRE), Luzern.

Genoud, P. A., Ruiz, G. \& Gurtner, J.-L. (2009). Évolution de la motivation scolaire des adolescents: différences selon la filière et le genre. Revue Suisse des Sciences de l'Éducation, 31(2), 377-395.

Goodenow, C. \& Grady, K. E. (1993). The relationship of school belonging and friends' values to academic motivation among urban adolescent students. Journal of Experimental Education, 62(1), 60-71.

Gunderson, E. A., Ramirez, G., Levine, S. C. \& Beilock, S.L. (2012). The role of parents and teachers in the development of gender-related math attitudes. Sex Roles, 66(3), 153-166. 
Gurtner, J.-L. \& Genoud, P. A. (2006). Facteurs contextuels dans l'évolution de la motivation pour le travail scolaire au cours de l'adolescence. In B. Galand \& E. Bourgeois (Éd.), (Se) motiver à apprendre (pp. 115-124). Paris: Presses Universitaires de France.

Kane, J. M. \& Mertz, J. (2012). Debunking myths about gender and mathematics performance. Notices of the American Mathematical Society, 59(1), 10-21.

Koller, P. \& Meffre, V. (2013). Les titulaires d'un diplôme MINT sur le marché du travail. Enquête auprès des personnes diplômées des hautes écoles: domaines mathématiques, informatique, sciences naturelles et technique. Neuchâtel: Office Fédéral de la Statistique (OFS).

Krohne, H. W. (2003). Individual differences in emotional reactions and coping. In R.J. Davidson, K. R. Scherer \& H. H. Goldsmith (Ed.), Handbook of Affective Sciences (pp. 698-725). Oxford: Oxford University Press.

Lafontaine, D. \& Monseur, C. (2009). Les évaluations des performances en mathématiques sont-elles influencées par le sexe de l'élève? Mesure et Évaluation en Éducation, 32(2), 71-98.

Lane, K. A., Goh, J. X. \& Driver-Linn, E. (2012). Implicit science stereotypes mediate the relationship between gender and academic participation. Sex Roles, 66(3-4), 220-234.

Leaper, C., Farkas, T. \& Brown, C.S. (2012). Adolescent girls' experiences and gender-related beliefs in relation to their motivation in math/science and English. Journal of Youth and Adolescence, 41(3), 268-282.

Lebarbier, E. \& Mary-Huard, T. (2006). Une introduction au critère BIC: Fondements théoriques et interprétation. Journal de la Société Française de Statistique, 147(1), 39-57.

Lichtenfeld, S., Pekrun, R., Stupnisky, R. H., Reiss, K. \& Murayama, K. (2012). Measuring students' emotions in the early years: The Achievement Emotions Questionnaire-Elementary School (AEQ-ES). Learning and Individual Differences, 22(2), 190-201.

Lindberg, S. M., Hyde, J. S., Petersen, J. L. \& Linn, M. C. (2010). New trends in gender and mathematics performance: a meta-analysis. Psychological Bulletin, 136(6), 1123-1135.

Ma, X. \& Kishor, N. (1997). Attitude toward self, social factors, and achievement in mathematics: A meta-analytic review. Educational Psychology Review, 9(2), 89-120.

Ma, X. \& Xu, J. (2004). Determining the causal ordering between attitude toward mathematics and achievement in mathematics. American Journal of Education, 110(3), 256-280.

Malmberg, L.-E. \& Little, T. D. (2007). Profiles of ability, effort, and difficulty: Relationships with worldviews, motivation and adjustment. Learning and Instruction, 17(6), 739-754.

Mariotta, M. (2011). Résultats en mathématiques. In Consortium PISA.ch (Éd), PISA 2009: Résultats régionaux et cantonaux (pp. 37-43). Berne et Neuchâtel: OFFT/CDIP et Consortium PISA.ch.

Marsh, H. W., Abduljabbar, A. S., Abu-Hilal, M. M., Morin, A. J., Abdelfattah, F., Leung, K. C. \& Parker, P. (2013). Factorial, convergent, and discriminant validity of TIMSS math and science motivation measures: A comparison of Arab and Anglo-Saxon countries. Journal of Educational Psychology, 105(1), 108-128.

Marsh, H. W., Martin, A. J. \& Cheng, J. H. (2008). A multilevel perspective on gender in classroom motivation and climate: Potential benefits of male teachers for boys? Journal of Educational Psychology, 100(1), 78-95.

Marsh, H. W. \& Yeung, A. S. (1998). Longitudinal structural equation models of academic self-concept and achievement: Gender differences in the development of math and English constructs. American Educational Research Journal, 35(4), 705-738.

Martin, A. J. (2004). School motivation of boys and girls: Differences of degree, differences of kind, or both? Australian Journal of Psychology, 56(3), 133-146.

Martinot, D., Bagès, C. \& Désert, M. (2012). French children's awareness of gender stereotypes about mathematics and reading: When girls improve their reputation in math. Sex Roles, 66(3-4), 210-219.

Milligan, G. W. (1980). An examination of the effect of six types of error perturbation on fifteen clustering algorithms. Psychometrika, 45(3), 325-342. 
Nagy, G., Watt, H. M., Eccles, J. S., Trautwein, U., Lüdtke, O. \& Baumert, J. (2010). The development of students' mathematics self-concept in relation to gender: Different countries, different trajectories? Journal of Research on Adolescence, 20(2), 482-506.

Nolen-Hoeksema, S. (2012). Emotion regulation and psychopathology: The role of gender. Annual Review of Clinical Psychology, 8, 161-187.

Nosek, B. A., Smyth, F. L., Sriram, N., Lindner, N. M., Devos, T., Ayala, A. \& al. (2009). National differences in gender-science stereotypes predict national sex differences in science and math achievement. Proceedings of the National Academy of Sciences, 106(26), 10593-10597.

Ntamakiliro, L., Monnard, I. \& Gurtner, J.-L. (2000). Mesure de la motivation scolaire des adolescents: construction et validation de trois échelles complémentaires. Orientation Scolaire et Professionnelle, 29(4), 673-693.

Op't Eynde, P., De Corte, E. \& Verschaffel, L. (2001). "What to learn from what we feel?": The role of students' emotions in the mathematics classroom. In S. Volet \& S. Järvelä (Ed.), Motivation in learning contexts: Theorical advances and methodological implications (pp. 149-167). Oxford: Pergamon.

Patrick, H., Mantzicopoulos, P., Samarapungavan, A. \& French, B. F. (2008). Patterns of young children's motivation for science and teacher-child relationships. Journal of Experimental Education, 76(2), 121-144.

Pekrun, R. (2006). The control-value theory of achievement emotions: Assumptions, corollaries, and implications for educational research and practice. Educational Psychology Review, 18(4), 315-341.

Pekrun, R., Elliot, A. J. \& Maier, M. A. (2009). Achievement goals and achievement emotions: Testing a model of their joint relations with academic performance. Journal of Educational Psychology, 101(1), 115-135.

Pekrun, R., Goetz, T., Frenzel, A. C., Barchfeld, P. \& Perry, R. P. (2011). Measuring emotions in students' learning and performance: The Achievement Emotions Questionnaire (AEQ). Contemporary Educational Psychology, 36(1), 36-48.

Plante, I., de la Sablonnière, R., Aronson, J.M. \& Théorêt, M. (2013). Gender stereotype endorsement and achievement-related outcomes: The role of competence beliefs and task values. Contemporary Educational Psychology, 38(3), 225-235.

Raynal, F. \& Rieunier, A. (2001). Pédagogie: dictionnaire des concepts clés (3e éd.). Paris: ESF. Ryan, A. M. (2001). The peer group as a context for the development of young adolescent motivation and achievement. Child Development, 72(4), 1135-1150.

Ryan, R. M. \& Deci, E. L. (2000). Intrinsic and extrinsic motivations: classic definitions and new directions. Contemporary Educational Psychology, 25(1), 54-67.

Sadler, P. M., Sonnert, G., Hazari, Z. \& Tai, R. (2012). Stability and volatility of STEM career interest in high school: A gender study. Science Education, 96(3), 411-427.

Simons, J., Dewitte, S. \& Lens, W. (2004). The role of different types of instrumentality in motivation, study strategies, and performance: Know why you learn, so you'll know what you learn! British Journal of Educational Psychology, 74(3), 343-360.

Smith, K. J., Davy, J. A. \& Rosenberg, D. L. (2012). An empirical analysis of an alternative configuration of the Academic Motivation Scale. Assessment in Education: Principles, Policy \& Practice, 19(2), 231-250.

Spencer, S. J., Steele, C. M. \& Quinn, D. M. (1999). Stereotype threat and women's math performance. Journal of Experimental Social Psychology, 35(1), 4-28.

Steinley, D. \& Brusco, M.J. (2011). Choosing the number of clusters in K-means clustering. Psychological Methods, 16(3), 285-297.

Stetsenko, A., Little, T. D., Gordeeva, T., Grasshof, M. \& Oettingen, G. (2000). Gender effects in children's beliefs about school performance: A cross-cultural study. Child Development, 71(2), 517-527. 
Stoet, G. \& Geary, D. C. (2013). Sex differences in mathematics and reading achievement are inversely related: within- and across-nation assessment of 10 years of PISA data. PLoS ONE, 8(3), e 57988.

Triandis, H. C. (1971). Attitude and attitude change. New York: John Wiley \& Sons.

Watt, H. M. (2004). Development of adolescents' self-perceptions, values, and task perceptions according to gender and domain in 7 th- through 11th-grade Australian students. Child Development, 75(5), 1556 - 1574.

Watt, H. M. (2006). The role of motivation in gendered educational and occupational trajectories related to maths. Educational Research and Evaluation: An International Journal on Theory and Practice, 12(4), 305-322.

Weiner, B. (1985). An attributional theory of achievement motivation and emotion. Psychological Review, 92(4), 548-573.

Wigfield, A., Eccles, J. S., Mac Iver, D., Reuman, D. A. \& Midgley, C. (1991). Transitions during early adolescence: Changes in children's domain-specific self-perceptions and general self-esteem across the transition to junior high school. Developmental Psychology, $27(4), 552-565$.

Wolters, C. A. \& Pintrich, P. R. (1998). Contextual differences in student motivation and self-regulated learning in mathematics, english, and social studies classrooms. Instructional Science, 26, 27-47.

Wyss, V. L., Huelskamp, D. \& Siebert, C. J. (2012). Increasing middle school student interest in STEM careers with videos of scientists. International Journal of Environmental and Science Education, 7(4), 501-522.

Mots-clés: Attitudes, mathématiques, adolescent, différences genre

\section{Haltung gegenüber Mathematik: haben Mädchen und Jungen die gleichen Einstellungen?}

\section{Zusammenfassung}

Dieser Artikel befasst sich mit der Analyse der affektiven und sozialen Einstellungen von Mädchen und Jungen zu ihren Lernprozessen in Mathematik während ihrer Gymnasialschulzeit. Mithilfe eines Selbsteinschätzungsfragebogens wurden die Wahrnehmungen des Fachs (wahrgenommener Nutzen, Kompetenzerleben, Kontrollierbarkeit) und affektive Erfahrungen (positiv, negativ und Gefühl der Selbstregulation) erfasst, sowie das Vorhandensein des Stereotyps der Männlichkeit bezüglich der Disziplin erhoben. Es konnten sechs charakteristische Profile ermittelt werden. Diese Profile sind nicht nur bezüglich Geschlecht verschieden verteilt, sondern verweisen auch auf Differenzen im erbrachten zeitlichen Lernaufwand. Die vorliegenden Resultate beleuchten den Einfluss affektiver und sozialer Einstellungen auf die Motivation, die Einschätzung schulischer Leistungen und Entscheidungen bei der Berufswahl bei Jugendlichen, die sich im Übergang von der Sekundarstufe II zu einer tertiären Hochschulbildung befinden.

Schlagworte: Haltungen, Mathematik, Jugendalter, Gender. 


\section{Attitudini verso la matematica: le ragazze e i ragazzi l'affrontano nello stesso modo?}

\section{Riassunto}

Questo articolo propone un'analisi delle attitudini socio-affettive dei ragazzi riguardo al loro apprendimento della matematica durante la scuola post-obbligatoria (liceo). Mediante un questionario autocompilato che valuta al tempo stesso le percezioni degli allievi di questa materia (utilità percepita, senso di competenza, controllabilità), i loro sentimenti (positivi, negativi, nonché la capacità di regolarli) e la presenza di stereotipi di mascolinità in matematica, abbiamo potuto evidenziare sei diversi profili. Questi ultimi non sono solamente ripartiti inegualmente secondo il genere, ma presentano delle differenze in termini di investimento nello studio. Tali risultati fanno riflettere sull'impatto delle attitudini sulla motivazione e sui risultati degli allievi (in particolare l'instaurazione di circoli viziosi), ma soprattutto a proposito della scelta d'orientamento professionale degli adolescenti che stanno per entrare in altre scuole superiori/ professionali.

Parole chiave: Attitudini, matematica, adolescente, diversità di genere

\section{Attitudes towards mathematics: are girls and boys equal in their approach to learning?}

\section{Abstract}

This article provides an analysis of socio-emotional attitudes of girls and boys concerning learning in mathematics during their post-compulsory education (high school). We were able to identify six typical profiles through a self-reported questionnaire assessing different perceptions students have in this discipline (perceived usefulness, perceived competence, controllability), their affect (positive, negative, and the feeling of being able to regulate) as well as the presence of the stereotype that math is associated to masculinity. These students are not only unequally distributed by gender but show differences in terms of investment. Such results question the impact of attitudes on motivation and student performance (including the vicious circles that are established), and especially on career guidance for teenagers about to enter universities and colleges.

Keywords: Attitudes, mathematics, teenager, gender differences 
